\title{
La gestión de programas académicos desde la perspectiva de la gestión del conocimiento apoyada con esquemas preconceptuales ${ }^{*}$
}

\author{
Héctor Jairo Ortiz Pabón ${ }^{* *}$ \\ Carlos Mario Zapata Jaramillo** \\ Guillermo González Calderón ${ }^{* * * *}$
}

Recibido: 02/21/2014 - Aceptado: 27/06/2014

\begin{abstract}
Resumen
Los procesos que se suelen asociar con los programas académicos y los objetivos organizacionales que los respaldan aún no se unifican en la literatura especializada. Esa unificación se puede lograr empleando procesos de gestión del conocimiento. Por ello, en este artículo se propone una ampliación a una versión previa del esquema preconceptual que representa la gestión de programas académicos, tomando en consideración la opinión de expertos mediante una encuesta diseñada para tal fin. El esquema resultante se ejemplifica con el programa de Ingeniería de Sistemas de la Universidad de Medellín.
\end{abstract}

Palabras clave: gestión del conocimiento, gestión de programas académicos, esquemas preconceptuales

* Artículo resultado del proyecto de investigación "Modelo de gestión del conocimiento en el Programa de Ingeniería de Sistemas de la Universidad de Medellín”, financiado por la Universidad de Medellín.

** Ingeniero de Sistemas, especialista en Gerencia de Información. M. Sc en Gestión de la Información y el Conocimiento. Correo: hortiz@udem.edu.co

*** Ingeniero Civil. Ph.D en Ingeniería. Profesor asociado de la Universidad Nacional de Colombia. Líder del grupo de investigación en Lenguajes Computacionales. Correo: cmzapata@unal.edu.co. Teléfono: (57)(4) 4255374. Fax: (57)(4) 4255365. Carrera 80 No. 65-223 Bloque M8A-310, Facultad de Minas, Escuela de Sistemas

**** M.Sc Ingeniería de Sistemas. Candidado a Doctor en Ingeniería, Universidad Nacional de Colombia. Correo: ggonzal@unal.edu.co 


\title{
Academic programs management from the perspective of knowledge management supported with pre-conceptual schemas
}

\begin{abstract}
Unification of academic program processes and goals is still far from the state of the art. Such unification can be reached by using knowledge management processes. For this reason, in this paper we propose a revisited version of a previous pre-conceptual-schema-based representation of the academic programs. We design a survey directed to experts and we obtain several responses. The resulting pre-conceptual schema is exemplified by using the system engineering program belonging to the Universidad de Medellín.
\end{abstract}

Key words: Knowledge management, academic program management, pre-conceptual schemas 


\section{INTRODUCCIÓN}

En un trabajo previo del grupo de investigación [1] se establecieron las principales dificultades asociadas con la gestión de programas académicos. En general, los procesos y objetivos se encuentran dispersos en diferentes enfoques [2-13], pero aun así se encuentran vacíos al comparar con los programas académicos reales y confrontar la opinión de gestores de esos programas.

Como en el trabajo previo [1], la gestión del conocimiento posibilita la apropiación de los conceptos de un determinado dominio para facilitar el análisis de los diferentes obstáculos que se afrontan allí y, consecuentemente, la toma de decisiones encaminada al mejoramiento de las organizaciones. Se pretende, de esta manera, dotar al gestor de programas académicos con una herramienta que facilite su labor y le suministre una visión holística del proceso.

Como continuidad al esquema preconceptual que se construyó previamente para representar la gestión de programas académicos [1], en este artículo se propone una ampliación que tome en consideración la opinión de gestores académicos reales que contribuyan a llenar los vacíos de información que se presentan en la literatura. Con este fin, se diseña una encuesta para su aplicación a diferentes expertos, cuyas respuestas se incorporan luego en forma de conceptos, relaciones dinámicas, instancias de conceptos y relaciones de logro en el esquema preconceptual existente. El esquema resultante se ejemplifica luego con diferentes documentos, procesos y situaciones que rodean la gestión del programa de Ingeniería de Sistemas de la Universidad de Medellín.

Este artículo se organiza de la siguiente manera: en la sección 2 se define el marco teórico correspondiente a la gestión del conocimiento y los esquemas preconceptuales; en la sección 3 se compendian los hallazgos previos para la construcción del esquema preconceptual base; en la sección 4 se propone el diseño de la encuesta, se compendian sus resultados y se amplía el esquema preconceptual base; en la sección 5 se plantea el caso de estudio con la información del programa de Ingeniería de Sistemas de la Universidad de Medellín; las conclusiones y el trabajo futuro se incluyen en la sección 6.

\section{MARCO TEÓRICO}

\section{GESTIÓN DEL CONOCIMIENTO}

King [14] define la gestión del conocimiento como "la planeación, organización, motivación y control de las personas, los procesos y los sistemas de una organización para asegurar que los activos relacionados con el conocimiento mejoren y se utilicen efectivamente". Además, King [14] establece que "los procesos de la gestión del conocimiento incluyen la adquisición, creación, refinamiento, almacenamiento, transferencia, 
compartición y uso del conocimiento", labor que realizan los encargados de la gestión del conocimiento en las diferentes organizaciones, quienes "operan estos procesos, desarrollan metodologías y sistemas para apoyar esos procesos y motivan a las personas a participar en ellos". Esta es la definición que mejor describe la metodología que se sigue en este artículo, si bien se procura el refinamiento de la información con base en la adquisición de nuevos datos y su incorporación en una representación previa de dicho conocimiento. La representación mejorada se puede emplear para el análisis del conocimiento, con miras a la toma de decisiones.

\section{ESQUEMAS PRECONCEPTUALES}

Zapata et al. [15] proponen los esquemas preconceptuales como representaciones del conocimiento relativas a un dominio específico. Se trata, pues, de acercar el conocimiento de un determinado dominio o área (que usualmente se escribe en lenguaje natural) al lenguaje simbólico (mediante una especie de grafo dirigido), específicamente para la obtención de esquemas conceptuales de UML. Sin embargo, los esquemas preconceptuales trascienden este uso y se pueden aplicar a diferentes áreas, en este caso a la gestión del conocimiento. Los principales símbolos que se emplean en los esquemas preconceptuales se muestran en la figura 1, incluyendo los elementos de los denominados esquemas preconceptuales ejecutables. Los conceptos incluyen sustantivos y sintagmas nominales; las relaciones estructurales incluyen los verbos ser y estar; las relaciones dinámicas contemplan los verbos de actividad; las relaciones de logro comprenden los verbos de objetivos (mejoramiento, mantenimiento y realización); las notas incluyen los posibles valores que se asocian con un concepto, y los marcos se emplean para agrupar las relaciones dinámicas en responsabilidades.

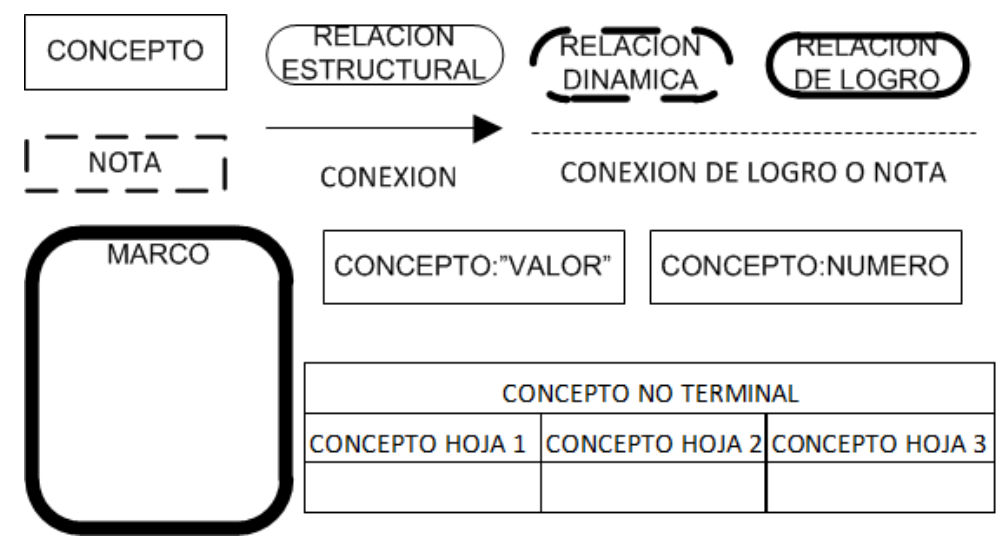

Figura 1. Símbolos que se emplean en los esquemas preconceptuales

Adaptación propia a partir de Zapata et al. [15] 
En el interior de un concepto, se puede ubicar información adicional sobre el valor que toma el concepto y en las tablas adicionales se puede detallar la información de los conceptos hoja (aquellos que reciben una relación del tipo "tiene" y de los que no parte ninguna relación), que pertenecen a conceptos no terminales (aquellos que no son conceptos hoja).

\section{TRABAJO PREVIO}

Zapata et al. [1] proponen una representación del conocimiento relativo a la gestión de programas académicos en instituciones de Educación Superior. Para ello, emplean el conocimiento que se encuentra disperso en diferentes artículos [2-13] y, luego de una revisión de contenidos, establecen una unificación terminológica que posibilita la comparación y consolidación de un esquema preconceptual común, que se muestra en la figura 2. Posteriormente, y tomando como base dicha figura, emplean tres esquemas preconceptuales ejecutables (véanse las figuras 3,4 y 5) para ejemplificar el uso del esquema de la figura 2, con miras a su uso en la gestión del conocimiento asociado con los programas académicos.

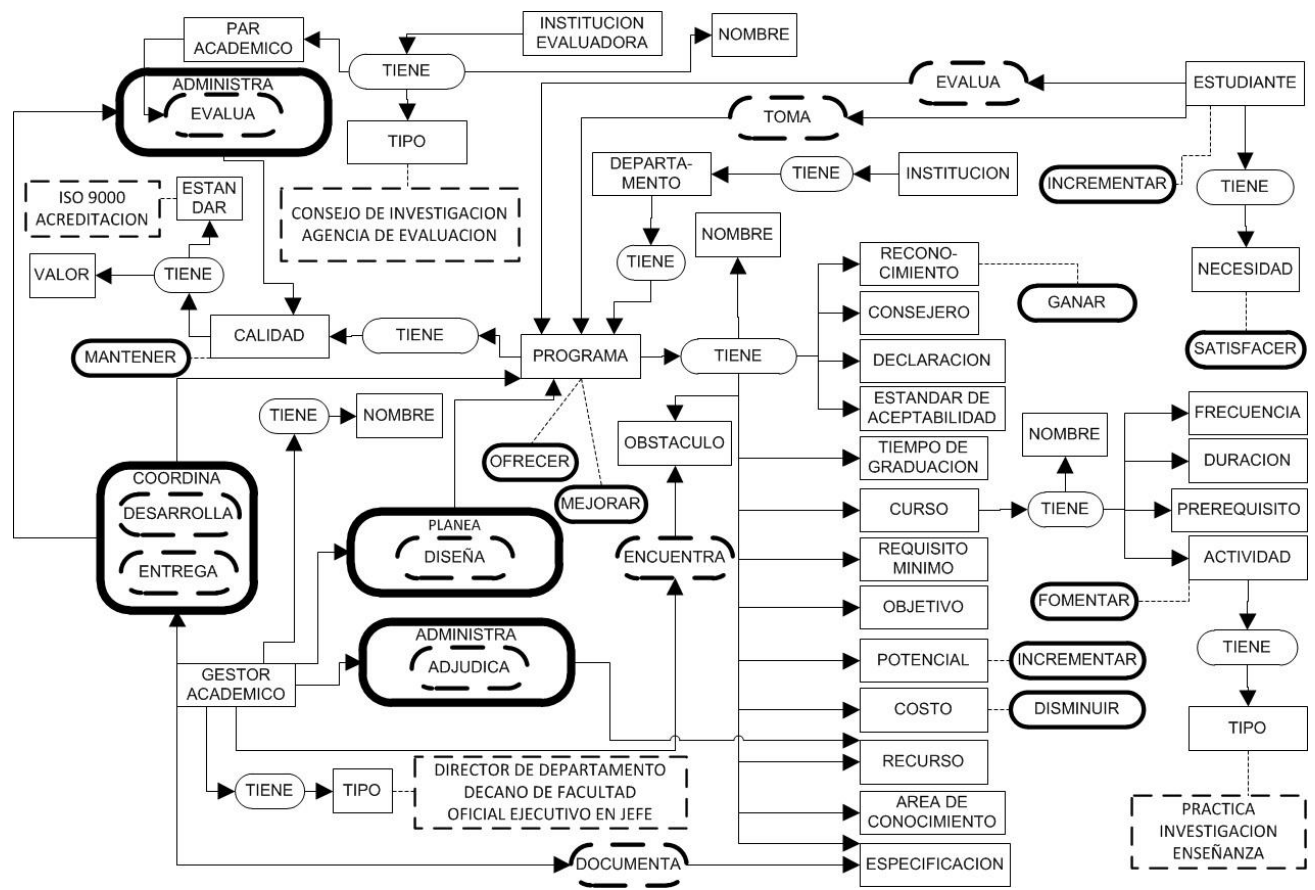

Figura 2. Esquema preconceptual que representa el conocimiento sobre la gestión de programas académicos en instituciones de Educación Superior.

Tomado de Zapata et al. [1] 


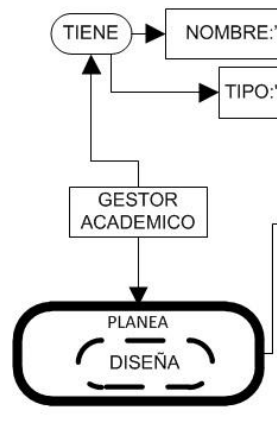

PO:"DIRECTOR DE DEPARTAMENTO"

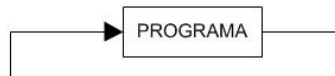

PROGRAMA

\begin{tabular}{|l|l|l|}
\hline INGENIERIA DE SISTEMAS & FUNDAMENTOS DE PROGRAMACION \\
\hline
\end{tabular}

INGENIERIA DE SISTEMAS LENGUAJES DE PROGRAMACION I \begin{tabular}{|l}
\hline INGENIERIA DE SISTEMAS LENGUAJES DE PROGRAMACION II \\
\hline
\end{tabular}

Figura 3. Esquema preconceptual ejecutable

para la relación dinámica "gestor académico diseña programa".

Tomado de Zapata et al. [1]

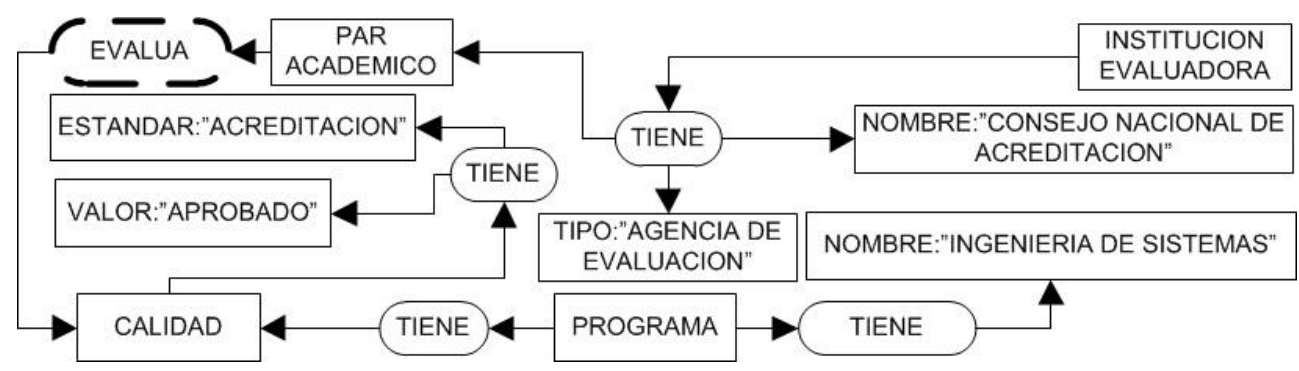

Figura 4. Esquema preconceptual ejecutable

para la relación dinámica "par académico evalúa calidad del programa"

Tomado de Zapata et al. [1]

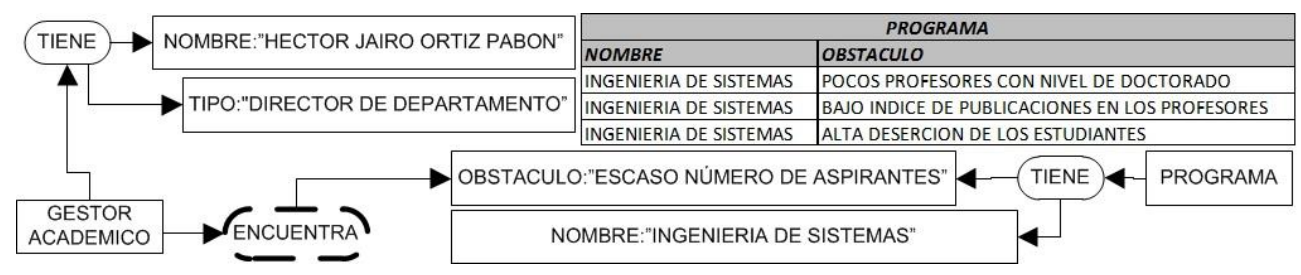

Figura 5. Esquema preconceptual ejecutable para la relación dinámica "gestor académico encuentra obstáculo"

Tomado de Zapata et al. [1]

\section{COMPLEMENTACIÓN DE LA REPRESENTACIÓN DEL CONOCIMIENTO SOBRE GESTIÓN DE PROGRAMAS ACADÉMICOS EN INSTITUCIONES DE EDUCACIÓN SUPERIOR}

Si bien la representación del conocimiento que se plantea en la figura 2 compendia los hallazgos que se pudieron establecer en la literatura especializada, la ejemplifica- 
ción muestra que aún existen ciertos vacíos asociados con ese conocimiento, lo que hace necesario el empleo de un mecanismo adicional para su complementación. En este caso, se opta por la consulta con gestores académicos de programas actualmente existentes para realizar esta labor, pues se considera que estos pueden aportar desde su experiencia la información necesaria para suplir los vacíos que se generan por el uso de la literatura especializada.

Para establecer la comunicación con los gestores académicos, se propone el diseño de una encuesta que incluya la representación del conocimiento de que se dispone, de forma tal que sirva, adicionalmente, como mecanismo de validación del conocimiento allí incluido. La encuesta diseñada es la siguiente:

\section{Encuesta para el mejoramiento del modelo de gestión del conocimiento de la Universidad de Medellín}

Nombre:

Programa:

Revise detenidamente el esquema de la figura 2, que representa la gestión del conocimiento de cualquier programa de pregrado de nuestra Universidad. Este esquema se elaboró tomando como base la revisión de literatura sobre el tema.

A partir de dicha información, ¿podría establecer otros elementos no incluidos en el esquema? Se sugiere la siguiente estructura:

1. Conceptos: se trata de sustantivos (por ejemplo CALIDAD) o sintagmas nominales (ESTÁNDAR DE ACEPTABILIDAD).

2. Acciones: se trata de verbos de actividad (por ejemplo, ENCUENTRA, DISEÑA, etc.)

3. Responsabilidades: se trata de verbos de acción más generales (por ejemplo, COORDINA, ADMINISTRA, etc.)

4. Objetivos: se trata de verbos de logro, clasificados en mejoramiento (por ejemplo, FOMENTAR, INCREMENTAR, etc.), mantenimiento (por ejemplo, CONSERVAR, MANTENER, etc.) y de realización (LOGRAR, HACER, etc.)

Tenga en cuenta que una respuesta viable puede ser que el esquema en cuestión representa adecuadamente la gestión de su programa y no requiere más información.

¿Puede proveer ejemplos de uso de los elementos que propone? Para tal efecto, en las figuras 3, 4 y 5 se incluyen otros esquemas que muestran el uso del diseño de programas, la evaluación de calidad y el encuentro de obstáculos. En este caso, los ejemplos pertenecen al área de Ingeniería de Sistemas, por ejemplo el curso de Análisis Geométrico, la evaluación del Consejo Nacional de Acreditación y el obstáculo "escaso número de aspirantes", pero sus ejemplos pueden ser de cualquier naturaleza sobre la información que quieren adicionar al modelo. 
La encuesta se aplicó a diez gestores académicos correspondientes a diferentes programas de universidades colombianas. En la tabla 1 se presentan las respuestas que suministraron los gestores académicos, junto con un análisis de cada respuesta para establecer los elementos que se deberían incorporar o modificar en el esquema preconceptual y la incidencia en dicho esquema. En opinión de los diez gestores académicos, el esquema de la figura 2 establece de forma adecuada el conocimiento relacionado con la gestión de programas académicos, pero faltan algunos elementos que se deducen, finalmente, de las respuestas. El único concepto que se modificó del esquema preconceptual de la figura 2 es "institución", que se cambió por el concepto "facultad" para expresar que los departamentos podrían o no estar adscritos a una determinada facultad. Finalmente, se incorporaron las adiciones sugeridas, que se resaltan en gris para poder diferenciarlas de la información original, y se obtiene el esquema preconceptual de la figura 6 .

Tabla 1. Análisis de las respuestas de los gestores académicos a la encuesta

\begin{tabular}{|c|c|c|}
\hline Respuesta & Análisis & $\begin{array}{c}\text { Incidencia en el } \\
\text { esquema preconceptual }\end{array}$ \\
\hline $\begin{array}{l}\text { En todos los programas y en es- } \\
\text { pecial los de ingeniería se debe } \\
\text { también gestionar políticas que } \\
\text { procuren la permanencia con ca- } \\
\text { lidad de los estudiantes, atacando } \\
\text { los factores relacionados con la de- } \\
\text { serción y promoviendo semilleros } \\
\text { académicos, programa sistemático } \\
\text { de tutorías, seminario permanente } \\
\text { de cualificación docente, semillero } \\
\text { de monitores, monitorias acadé- } \\
\text { micas, seguimiento y acompa- } \\
\text { ñamiento a becarios, diversidad } \\
\text { cultural e inclusión, curso de in- } \\
\text { ducción, asesoría y atención psi- } \\
\text { copedagógica y escritura de textos } \\
\text { (Ciencias Básicas, Universidad de } \\
\text { Medellín) }\end{array}$ & $\begin{array}{l}\text { Dentro de los RECURSOS, se pueden in- } \\
\text { cluir los elementos que se señalan en esta } \\
\text { respuesta. Además, es importante incluir } \\
\text { en el programa el índice de deserción, re- } \\
\text { formulando el objetivo de "permanencia } \\
\text { con calidad" como "disminuir deserción" }\end{array}$ & $\begin{array}{l}\text { Programa tiene índice de } \\
\text { deserción } \\
\text { Disminuir índice de } \\
\text { deserción } \\
\text { "Semilleros académicos", } \\
\text { "programa sistemático de } \\
\text { tutorías", "seminario per- } \\
\text { manente de cualificación } \\
\text { docente", "semillero de } \\
\text { monitores", "monitorias } \\
\text { académicas", "segui- } \\
\text { miento y acompañamien- } \\
\text { to a becarios", "diversi- } \\
\text { dad cultural e inclusión", } \\
\text { "curso de inducción", } \\
\text { "asesoría y atención psi- } \\
\text { copedagógica"y "escritu- } \\
\text { ra de textos" son posibles } \\
\text { valores de recurso }\end{array}$ \\
\hline $\begin{array}{l}\text { No solamente es docencia; hay } \\
\text { que fomentar la investigación, } \\
\text { extensión e internacionalización. } \\
\text { (Ingeniería Ambiental, Universi- } \\
\text { dad de Medellín) }\end{array}$ & $\begin{array}{l}\text { La docencia se representa con el con- } \\
\text { cepto "programa". Se deben incluir la } \\
\text { investigación, la extensión y la inter- } \\
\text { nacionalización en cabeza del departa- } \\
\text { mento y se deben incluir los objetivos } \\
\text { correspondientes. }\end{array}$ & $\begin{array}{l}\text { Departamento tiene in- } \\
\text { vestigación, extensión e } \\
\text { internacionalización } \\
\text { Fomentar investigación } \\
\text { Fomentar extensión } \\
\text { Fomentar investigación }\end{array}$ \\
\hline
\end{tabular}




\begin{tabular}{|c|c|c|}
\hline Respuesta & Análisis & $\begin{array}{c}\text { Incidencia en el } \\
\text { esquema preconceptual }\end{array}$ \\
\hline $\begin{array}{l}\text { Aparte de la coordinación de las } \\
\text { otras funciones sustantivas de la } \\
\text { academia (investigación y exten- } \\
\text { sión) ya que la internacionaliza- } \\
\text { ción las permea a todas, hay que } \\
\text { lograr la mayor retención posible } \\
\text { del número de estudiantes redu- } \\
\text { ciendo la deserción. Adicional- } \\
\text { mente, en el caso de la Universidad } \\
\text { de Medellín, los programas están } \\
\text { adscritos a facultades, no a depar- } \\
\text { tamentos (Ingeniería de Sistemas, } \\
\text { Universidad de Medellín) }\end{array}$ & $\begin{array}{l}\text { Se refuerza en esta respuesta la inclu- } \\
\text { sión de la investigación, la extensión y } \\
\text { la internacionalización, además de la } \\
\text { inclusión del índice de deserción. Para } \\
\text { establecer el caso general, se incluirá } \\
\text { el concepto "facultad", que incluye los } \\
\text { "departamentos". En caso de que los de- } \\
\text { partamentos no existan, por transitividad } \\
\text { los programas pasan a las facultades. }\end{array}$ & $\begin{array}{l}\text { F a c u l t a d tie n e } \\
\text { departamento }\end{array}$ \\
\hline $\begin{array}{l}\text { Debe diseñar estrategias que per- } \\
\text { mitan volver el programa compe- } \\
\text { titivo (no solo gestión académica, } \\
\text { incluir la gestión de capacitaciones } \\
\text { y certificaciones internacionales } \\
\text { para sus estudiantes y egresados). } \\
\text { (Ingeniería de Telecomunicacio- } \\
\text { nes, Universidad de Medellín) }\end{array}$ & $\begin{array}{l}\text { La gestión académica se representa ya en } \\
\text { el esquema con las diferentes actividades } \\
\text { relacionadas con el programa. Se refuer- } \\
\text { za la responsabilidad de administración } \\
\text { de los recursos y la inclusión de capaci- } \\
\text { taciones como el seminario permanente } \\
\text { de cualificación docente. Aparecen como } \\
\text { recurso, también, las certificaciones } \\
\text { internacionales }\end{array}$ & $\begin{array}{l}\text { "Certificación interna- } \\
\text { cional para estudiantes y } \\
\text { egresados" es un posible } \\
\text { valor de recurso }\end{array}$ \\
\hline $\begin{array}{l}\text { La administración de un programa } \\
\text { académico, incluye todo lo rela- } \\
\text { cionado con evaluaciones, segui- } \\
\text { miento y atención a estudiantes, } \\
\text { programación académica, semille- } \\
\text { ros de investigación. (Mercadeo, } \\
\text { Universidad de Medellín) }\end{array}$ & $\begin{array}{l}\text { Las evaluaciones y el seguimiento se } \\
\text { incluyen en una nueva actividad llamada } \\
\text { "controla programa", que está dentro de la } \\
\text { responsabilidad de coordinar programa. } \\
\text { La programación académica ya se tomó } \\
\text { en cuenta en el esquema en la responsabi- } \\
\text { lidad "planea programa". Los semilleros } \\
\text { de investigación y la atención a estudian- } \\
\text { tes se incluyen como otros recursos }\end{array}$ & $\begin{array}{l}\text { Gestor académico contro- } \\
\text { la programa } \\
\text { "Semillero de investiga- } \\
\text { ción" y "atención a es- } \\
\text { tudiantes" son posibles } \\
\text { valores de recurso }\end{array}$ \\
\hline $\begin{array}{l}\text { Un Programa también tiene Mi- } \\
\text { sión, Visión, Trabajo de Grado } \\
\text { (que a su vez tiene distintas mo- } \\
\text { dalidades) y Plan de educación de } \\
\text { Programa (PEP). También consi- } \\
\text { dero que si se trata de la gestión del } \\
\text { conocimiento se debería tener en } \\
\text { cuenta la(s) línea de investigación } \\
\text { del Programa con sus recursos } \\
\text { (semilleros, grupos, etc.). (Inge- } \\
\text { niería Informática, Corporación } \\
\text { Universitaria Lasallista, Medellín) }\end{array}$ & $\begin{array}{l}\text { De esta respuesta, se deben incluir la } \\
\text { misión, la visión, las modalidades de } \\
\text { trabajo de grado y el plan de educación } \\
\text { del programa. Aparecen los grupos al } \\
\text { interior de la investigación de los depar- } \\
\text { tamentos. Los semilleros se incluyeron } \\
\text { como recursos en una de las respuestas } \\
\text { iniciales. }\end{array}$ & $\begin{array}{l}\text { Programa tiene misión, } \\
\text { visión, modalidad de tra- } \\
\text { bajo de grado y plan de } \\
\text { educación pep } \\
\text { Investigación tiene grupo }\end{array}$ \\
\hline
\end{tabular}




\begin{tabular}{|c|c|c|}
\hline Respuesta & Análisis & $\begin{array}{c}\text { Incidencia en el } \\
\text { esquema preconceptual }\end{array}$ \\
\hline $\begin{array}{l}\text { Es muy importante que en el mo- } \\
\text { delo se refleje que la coordinación } \\
\text { de un programa académico incluye } \\
\text { hacer todos los calendarios acadé- } \\
\text { micos, y evaluación y seguimien- } \\
\text { to a los profesores, estudiantes y } \\
\text { monitores buscando incrementar } \\
\text { la calidad de todos los procesos } \\
\text { académicos. (Ingeniería de Siste- } \\
\text { mas, Institución Universitaria de } \\
\text { Envigado) }\end{array}$ & $\begin{array}{l}\text { Todos los elementos de esta respuesta ya } \\
\text { se incluyeron previamente. }\end{array}$ & \\
\hline $\begin{array}{l}\text { No sé si el concepto "Departamen- } \\
\text { to" aplique en todos los casos. En } \\
\text { mi institución los programas están } \\
\text { adscritos a las facultades y no a los } \\
\text { departamentos. } \\
\text { Puede ser valioso mostrar cómo } \\
\text { los docentes dan clases en un pro- } \\
\text { grama. Puede ser que no se asocien } \\
\text { directamente a los cursos, pero } \\
\text { pueden estar asociados al mismo } \\
\text { programa o a otras unidades (como } \\
\text { los departamentos que te comenta- } \\
\text { ba), y de ellos se puede guardar su } \\
\text { título y áreas de interés. } \\
\text { Si se trata de un modelo pensando } \\
\text { en evaluaciones (ya sea internas o } \\
\text { externas), sería importante incluir } \\
\text { factores o indicadores o caracte- } \\
\text { rísticas (en general, elementos de } \\
\text { medición) que el programa desea } \\
\text { evaluar, incluyendo su descrip- } \\
\text { ción, forma de medir y resultado. } \\
\text { También dependiendo de lo que } \\
\text { se desee con este modelo, pienso } \\
\text { que puede incluirse la información } \\
\text { adicional que desarrolle un progra- } \\
\text { ma (por fuera de los cursos), como } \\
\text { semilleros de investigación, ferias, } \\
\text { intercambios, entre otras. (Inge- } \\
\text { niería de Sistemas, Universidad } \\
\text { Autónoma de Manizales) }\end{array}$ & $\begin{array}{l}\text { La duda sobre los departamentos se } \\
\text { resolvió al incluir las Facultades. } \\
\text { Aparece el concepto "profesor" adscrito } \\
\text { al departamento, incluyendo sus títulos, } \\
\text { áreas de interés y cursos. Los tópicos re- } \\
\text { lativos a la evaluación ya se contemplan } \\
\text { en el esquema, pero es importante incluir } \\
\text { los factores y los indicadores dentro de } \\
\text { la calidad, pues de esta forma se puede } \\
\text { definir un esquema de medición. } \\
\text { Aparecen, también, ferias e intercambios } \\
\text { como recursos del programa. }\end{array}$ & $\begin{array}{l}\text { Departamento tiene } \\
\text { profesor } \\
\text { Profesor tiene título, área } \\
\text { de interés y curso } \\
\text { Calidad tiene factor e } \\
\text { indicador } \\
\text { "Ferias" e "intercambios" } \\
\text { son posibles valores de } \\
\text { recursos }\end{array}$ \\
\hline
\end{tabular}


La gestión de programas académicos desde la perspectiva de la gestión del conocimiento apoyada con ... 201

\begin{tabular}{|c|c|c|}
\hline Respuesta & Análisis & $\begin{array}{c}\text { Incidencia en el } \\
\text { esquema preconceptual }\end{array}$ \\
\hline $\begin{array}{l}\text { Respetuosamente, me permito } \\
\text { hacer los siguientes aportes que } \\
\text { hacen parte de nuestro plan de } \\
\text { mejoramiento continuo y, por } \\
\text { ende, de nuestros planes de ac- } \\
\text { ción. Diagramar Ejes estratégicos } \\
\text { como: Docencia, investigación, } \\
\text { extensión y proyección, egresados, } \\
\text { infraestructura física y tecnológi- } \\
\text { ca, gestión de la organización. (In- } \\
\text { geniería de Sistemas, Universidad } \\
\text { Cooperativa de Colombia, Ibagué) }\end{array}$ & $\begin{array}{l}\text { Los diferentes ejes estratégicos a que se } \\
\text { refiere esta respuesta, ya se incluyeron } \\
\text { con la información de la presente tabla. } \\
\text { La gestión de la organización también } \\
\text { está incluida en el esquema en forma de } \\
\text { las diferentes responsabilidades. }\end{array}$ & \\
\hline $\begin{array}{l}\text { Coordinar las diferentes actuali- } \\
\text { zaciones a los microcurrículos de } \\
\text { acuerdo a las tecnologías de punta. } \\
\text { Conservar la calidad del programa } \\
\text { fomentando la extensión e inves- } \\
\text { tigación. (Ingeniería de Sistemas, } \\
\text { Universidad de la Costa CUC, } \\
\text { Barranquilla) }\end{array}$ & $\begin{array}{l}\text { Dentro de la responsabilidad "planea } \\
\text { programa" se debe incluir una actividad } \\
\text { "actualiza programa". Los objetivos de } \\
\text { calidad a que se refiere esta respuesta se } \\
\text { tuvieron ya en cuenta. }\end{array}$ & $\begin{array}{l}\text { Gestor académico actua- } \\
\text { liza programa }\end{array}$ \\
\hline
\end{tabular}

Fuente: elaboración propia

Como resultado general del análisis del esquema preconceptual de la figura 6, se puede concluir que las principales responsabilidades del gestor académico son:

- Planear el programa incluyendo su diseño y actualización.

- La coordinación del programa en temas de docencia, investigación, extensión e internacionalización.

- La administración de los recursos del programa.

- La administración de la calidad del programa.

Además, el gestor responde por el encuentro de los obstáculos del programa y la documentación de especificaciones. Los objetivos asociados con la labor de los gestores académicos son:

- $\quad$ El incremento en el número de estudiantes.

- $\quad$ La satisfacción de las necesidades de los estudiantes.

- La disminución del índice de deserción del programa.

- La disminución de los costos del programa. 


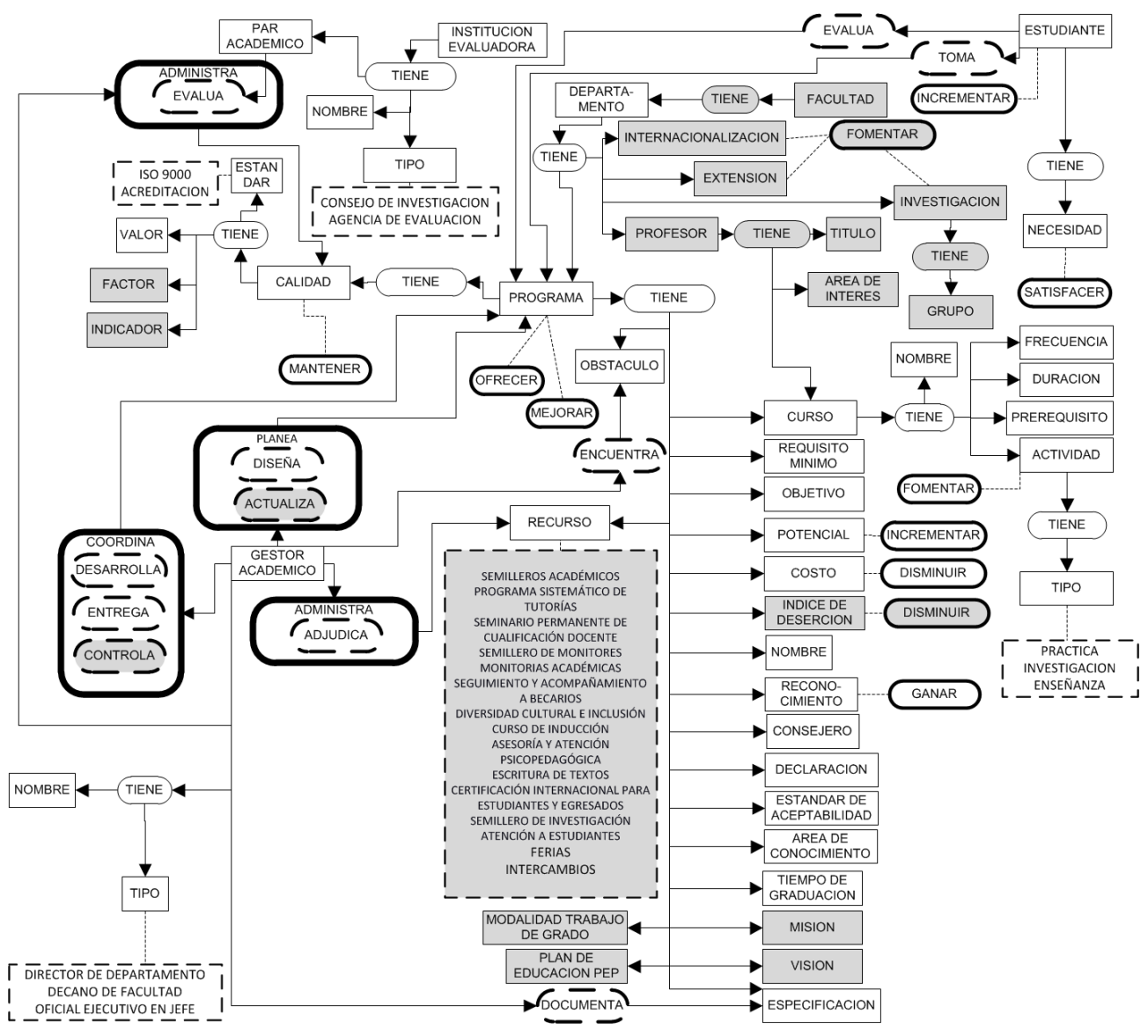

Figura 6. Esquema preconceptual complementado con la información de los gestores académicos Fuente: elaboración propia

- $\quad$ El incremento del potencial del programa.

- La ganancia de reconocimiento del programa.

- $\quad$ El fomento de las actividades de los cursos.

- El fomento de la internacionalización, la extensión y la investigación.

- La mejora del programa.

- El mantenimiento de la calidad del programa.

- $\quad$ El ofrecimiento del programa.

\section{CASO DE ESTUDIO}

Tomando como base el esquema preconceptual de la figura 6, en esta sección se continúa con el análisis del programa de Ingeniería de Sistemas de la Universidad de 
Medellín. En este caso, la relación dinámica "gestor académico encuentra obstáculo" (véase la figura 5) se puede complementar con la información adicional que sugirieron los gestores académicos consultados, de forma tal que se incluya el recurso a que se dirige el obstáculo y el objetivo del programa que se afecta con el obstáculo, como se puede apreciar en la figura 7. Este análisis posibilita el estudio de las decisiones que permitan superar los obstáculos y lograr, finalmente, el cumplimiento de los objetivos del programa.

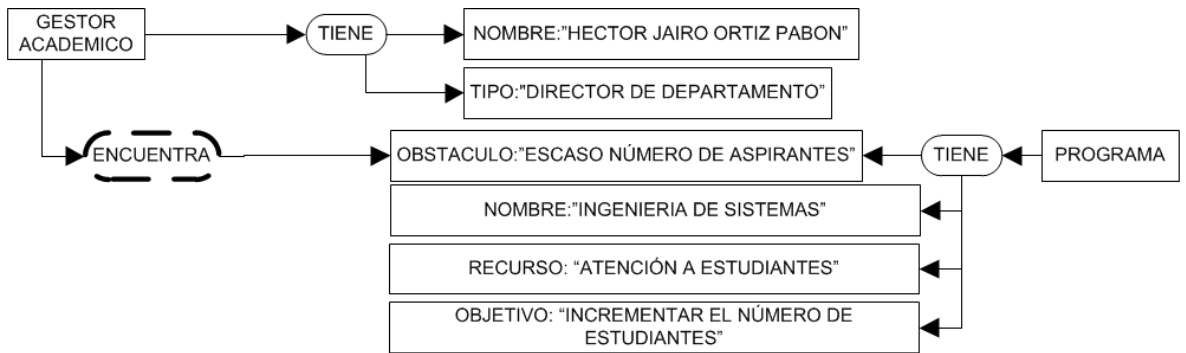

\begin{tabular}{l|l|l|l|}
\hline \multicolumn{2}{|c|}{ PROGRAMA } \\
\hline NOMBRE & OBSTACULO & RECURSO & OBJETIVO \\
\hline INGENIERIA DE SISTEMAS & POCOS PROFESORES CON NIVEL DE DOCTORADO & $\begin{array}{l}\text { SEMINARIO PERMANENTE DE } \\
\text { CUALIFICACIÓN DOCENTE }\end{array}$ & $\begin{array}{l}\text { GANAR RECONOCIMIENTO DEL } \\
\text { PROGRAMA }\end{array}$ \\
\hline INGENIERIA DE SISTEMAS & BAJO INDICE DE PUBLCACIONES EN LOS PROFESORES & ESCRITURA DE TEXTOS & FOMENTAR LA INVESTIGACIÓN \\
\hline INGENIERIA DE SISTEMAS & ALTA DESERCION DE LOS ESTUDIANTES & $\begin{array}{l}\text { ASESORÍA Y ATENCIÓN } \\
\text { PSICOPEDAGÓGICA }\end{array}$ & $\begin{array}{l}\text { DISMINUIR EL ÍNDICE DE DESERCIÓN } \\
\text { DEL PROGRAMA }\end{array}$ \\
\hline INGENIERIA DE SISTEMAS & $\begin{array}{l}\text { POCOS SISTEMAS DE INFORMACIÓN PARA APOYAR AL } \\
\text { GESTOR ACADÉMICO DEL PROGRAMA }\end{array}$ & $\begin{array}{l}\text { ATENCIÓN A ESTUDIANTES } \\
\text { DISMINUIR EL ÍNDICE DE DESERCIÓN } \\
\text { DEL PROGRAMA }\end{array}$ & $\begin{array}{l}\text { FOMENTAR LA } \\
\text { INTERNACIONALIZACIÓN }\end{array}$ \\
\hline INGENIERIA DE SISTEMAS & $\begin{array}{l}\text { FALTA DE UNA POLITICA DEFINIDA PARA EL APOYO A LA } \\
\text { ASISTENCIA A EVENTOS INTERNACIONALES }\end{array}$ & $\begin{array}{l}\text { SEMINARIO PERMANENTE DE } \\
\text { CUALIFICACIÓN DOCENTE }\end{array}$ & FOMENTAR LA INVESTIGACIÓN \\
\hline
\end{tabular}

Figura 7. Esquema preconceptual ejecutable para la relación dinámica "gestor académico encuentra obstáculo" tomando como base el esquema preconceptual ampliado

Fuente: elaboración propia

\section{CONCLUSIONES Y TRABAJO FUTURO}

En este artículo se propuso la ampliación del esquema preconceptual que representa el conocimiento relacionado con la gestión de programas académicos en instituciones de Educación Superior. Se tomaron, como base para la ampliación los resultados de un conjunto de entrevistas, gestores académicos en ejercicio, quienes revisaron el esquema preconceptual existente y propusieron la adición de conceptos, notas, relaciones estructurales, relaciones dinámicas y relaciones de logro.

En general, los gestores académicos encuestados manifestaron que el esquema preconceptual y los ejemplos que se les entregaron con la encuesta representaban adecuadamente la terminología y la estructura del dominio de la gestión académica de programas, pero establecieron otros elementos que complementaron dicho esquema. 
A partir del esquema mejorado fue posible realizar un análisis más intensivo de los obstáculos que se encontraron en el programa de Ingeniería de Sistemas de la Universidad de Medellín, con miras a su análisis intensivo que conduzca a soluciones adecuadas a cada uno de esos obstáculos. De esta manera, el esquema preconceptual resultante se convierte en una herramienta de gestión del conocimiento, al facilitar la toma de decisiones en relación con estos temas.

Las líneas de trabajo futuro que se pueden derivar de este trabajo son:

- Desarrollar un prototipo que posibilite la comprensión de los diferentes elementos asociados con el esquema preconceptual y que tome como base dicho esquema.

- Establecer otras formas de validación y complementación del esquema, tales como los inventarios de procesos, tecnologías y convenios de que pueda disponer el gestor académico de un programa.

- Complementar el esquema preconceptual con detalles de diferentes conceptos que no se abordaron ni en la revisión de la literatura ni en las encuestas a los gestores académicos. Por ejemplo, el manejo de los factores y los indicadores para establecer la calidad del programa tienen características muy definidas de acuerdo con el Consejo Nacional de Acreditación y se podrían incluir para complementar el análisis.

\section{REFERENCIAS}

[1] C. M. Zapata, H. Ortiz y G. González, "El gestor de programas académicos: una representación del conocimiento basada en esquemas preconceptuales", L. López (Editor), Ingeniería de Software e Ingeniería del Conocimiento: dos disciplinas interrelacionadas. Sello Editorial Universidad de Medellín, pp 363-376, Medellín, Colombia, 2013.

[2] M. Murray, "Modern Management Applied to Academic Decisions", The Academy of Management Review, vol. 1, n. ${ }^{\circ}$ 1, pp. 79-88, 1976.

[3] D. Bland, "Managing Higher Education", Cassell Educational, Londres, 1990.

[4] M. Clayton, "Towards Total Quality Management in Higher Education at Aston University: A Case Study”, Higher Education, vol. 25, n. 3, pp. 363-371, 1993.

[5] K. Butler, "Quality Assessment in the Delivery of Academic Programs", Computers in Industrial Engineering, Vol. 29, n. ${ }^{\circ}$ 1-4, pp. 71-75, 1995.

[6] T. Mech, "The Managerial Roles of Chief Academic Officers", The Journal of Higher Education, Vol. 68, n. ${ }^{\circ}$ 3, pp. 282-298, 1997.

[7] V. Meek y F. Wood, "Higher Education Governance and Management: an Australian study", Australian Government Publishing Service, Canberra, 1997. 
[8] P. Gumport, "Academic Restructuring: Organizational Change and Institutional Imperatives", Higher Education, Vol. 39, n. ${ }^{\circ}$ 1, pp. 67-91, 2000.

[9] S. Mizrahi y A. Mehrez, "Managing quality in higher education systems via minimal quality requirements: signaling and control", Economics of Education Review, Vol. 21, pp. 53-65, 2002.

[10] T. Baker, "Academic programs in North America”, J. Dyro (Editor), Clinical Engineering Handbook. Elsevier Academic Press, Burlington, pp. 287-293, 2004.

[11] F. Brunicardi, “Academic Program Development”, Journal of Surgical Research, Vol 128, n. ${ }^{\circ}$ 2, pp. 232-237, 2005.

[12] K. Booker y Ch. Hilgenberg, "Analysis of academic programs: comparing nursing and other university majors in the application of a quality, potential and cost model", Journal of Professional Nursing, Vol. 26, n. ${ }^{\circ}$, pp. 201-206, 2010.

[13] S. Abdullah, A. Latiff, Th. Paraidathathu, A. Jaafar, W. Wan Ahmad, S. Hussein y S. Daud, "Gap Analysis towards Harmonisation of the MQA Code of Practice for Programme Accreditation with the Quality Management System of MS ISO 9001:2008”, Procedia Social and Behavioral Sciences, Vol. 18, pp. 436-441, 2011.

[14] W. King, "Knowledge Management and Organizational Learning”, W. King (Editor), Knowledge Management and Organizational Learning. Springer, pp. 3-14, New York, USA, 2009.

[15] C. M. Zapata, A. Gelbukh y F. Arango, "Pre-conceptual Schemas: a conceptual-graph-like knowledge representation for requirements elicitation", Lecture Notes in Computer Science, Vol. 4293, pp. 17-27, 2006. 